\title{
Association between glycemic control and risk of venous thromboembolism in diabetic patients: $A$ nested case-control study
}

\section{Sarah H R Charlier ( $\nabla$ sarah.charlier@usb.ch )}

University of Basel: Universitat Basel https://orcid.org/0000-0002-0039-0630

\section{Christian Meier}

University Hospital Basel: Universitatsspital Basel

\section{Susan S Jick}

Boston Collaborative Drug Surveillance Program

\section{Christoph R Meier}

University of Basel: Universitat Basel

\section{Claudia Becker}

University of Basel: Universitat Basel

\section{Research Article}

Keywords: diabetes mellitus type 2, venous thromboembolism, VTE, glycemic control, HbA1c, sex, casecontrol study

Posted Date: October 1st, 2021

DOl: https://doi.org/10.21203/rs.3.rs-882567/v1

License: (1) This work is licensed under a Creative Commons Attribution 4.0 International License.

Read Full License

Version of Record: A version of this preprint was published at Cardiovascular Diabetology on January 4th, 2022. See the published version at https://doi.org/10.1186/s12933-021-01432-1. 


\section{Abstract}

Background:

Previous studies suggested an elevated risk of venous thromboembolism (VTE) among patients with type 2 diabetes mellitus (T2DM), with a possible sex difference. The impact of glycemic control on the risk of VTE is unclear. Our objective was to analyze the association between glycemic control and the risk of unprovoked (idiopathic) VTE in men and women with T2DM.

Methods:

We conducted a nested case-control analysis (1:4 matching) within a cohort of patients with incident T2DM between 1995-2019 using data from the CPRD GOLD. We excluded patients with known risk factors for VTE prior to onset of DM. Cases were T2DM patients with an unprovoked treated VTE. The exposure of interest was glycemic control measured as $\mathrm{HbA} 1 \mathrm{c}$ levels. We conducted conditional logistic regression analyses adjusted for several confounders.

Results:

We identified 3'896 VTE cases and 15'584 controls (56\% females). We found no association between the $\mathrm{HbA1c}$ level and the risk of VTE in our main analysis. However, when the most recent $\mathrm{HbA} 1 \mathrm{c}$ value was within 90 days before the index date, women with $\mathrm{HbA} 1 \mathrm{c}$ levels $>7.0 \%$ had an $18-35 \%$ increased relative risk of VTE when compared to women with $\mathrm{HbA} 1 \mathrm{c}>6.5-7.0 \%$.

Conclusions:

Our study raises the possibility that female T2DM patients with $\mathrm{HbA} 1 \mathrm{c}$ levels $>7 \%$ may have a slightly higher risk for unprovoked VTE compared to women with $\mathrm{HbA} 1 \mathrm{c}$ level $>6.5-7.0 \%$. This increase may not be causal and may reflect differences in life style or other characteristics. We observed no effect of glycemic control on the risk of VTE in men.

\section{Introduction}

Diabetes mellitus (DM) is a chronic disease with a high global prevalence, affecting some 450 million (8.8\%) patients worldwide and causing approximately 5 million deaths per year.[1] In the UK, 4.7 million patients (7.0\%) had diagnosed or undiagnosed DM in 2019.[2] Because the majority (90\%) of the cases are DM type 2 (T2DM) [2], T2DM and its complications are of great importance for the health system. [1] T2DM is characterized by hyperglycemia due to insulin deficiency and insulin resistance, and it is linked to an increased risk for several cardiovascular diseases.[3, 4]

While it has been shown that T2DM patients have a higher risk for arterial thrombosis, the association between T2DM and the risk of venous thromboembolism (VTE) has been studied less. VTE, a medical condition in which a thrombus forms in the venous system, can manifest as deep vein thrombosis (DVT) 
or as pulmonary embolism (PE), if the thrombus travels to the pulmonary arteries.[5-7] VTE is associated with a high mortality.[5, 8] Its prevention and management is a priority for the NHS.[9] Unprovoked VTE [5] occurs at an incidence of 62.1 per 100'000 person years.[10] However, especially at older ages (>60 years), men have an approximately $20-25 \%$ higher incidence rate of VTE than women.[7, 11] The term unprovoked is used in accordance with the definition provided by the NICE guideline on PE and DVT, meaning that - similar to the term idiopathic - no recent known major risk factors were present prior to the VTE.[5]

Published findings regarding DM as an independent risk factor for VTE are not consistent $[6,12]$ However, it is well established that VTE occurs more than twice as often in patients with DM than in DM-free individuals. $[13,14]$ Studies also show that men are at a higher risk for T2DM and VTE than women when both diseases are considered individually, while women are at higher risk of VTE once other comorbidities (such as DM, cardiovascular disease, and atherosclerosis) are involved.[12, 15, 16]

Since the degree of hyperglycemia is crucial in the development of DM-related complications, $[17,18]$ the question arises whether there is also an association between hyperglycemia and the risk of VTE.

To date, recent studies assessing the impact of glycemic control on the risk of VTE in male and female patients with DM yielded conflicting results. While some authors found a statistically significant association between the level of glycemic control and the risk of VTE,[19, 20] others did not.[21] In a population-based cohort study from Norway, the risk of VTE increased by $5 \%$ per one standard deviation increase in $\mathrm{HbA} 1 \mathrm{c}$. However, in this study, there were no HbA1c measurements available at a time point close to the VTE event.[21] None of the published studies analyzed the impact of glycemic control on the risk of VTE stratified by sex. However, the sex of the patient could not only have an impact on the development and progression of the disease itself,[15, 22-24] but also on the association of glycemic control and risk of VTE.

A hypothesized pathway for an increased risk of VTE in patients with DM is that hyperglycemia contributes to elevated coagulation factors and impaired fibrinolysis. $[4,13,25]$ A single unifying mechanism of DM complications might be hyperglycemia-induced overproduction of superoxide by the mitochondrial electron transport chain, which activates several damaging pathways.[26] The activation of these pathways causes additional intracellular oxidative stress, abnormalities of the gene expression of glomerular cells, hyperglycemia-induced cardiomyocyte dysfunction, and an increase of the enzyme GFAT (glutamine fructose- 6 phosphate amidotransferase), resulting in a variety of effects on gene expression and advanced glycation end product formation.[26]

The objective of the present study was therefore to analyze the association between glycemic control and the risk of unprovoked VTE in patients with T2DM overall, as well as separately for men and women.

\section{Methods}

\section{Study design and data source}


We conducted a nested case-control analysis within a cohort of patients with incident T2DM between 01. January 1995 and 31. December 2019 in the UK-based primary care Clinical Practice Research Datalink (CPRD) GOLD.

CPRD GOLD contains anonymized medical records of over 11.3 million patients from more than 600 general practices in the UK. It is a governmental, non-profit database; the enrolled patients account for approximately $6.9 \%$ of the UK population. Patients within CPRD GOLD are representative of the UK general population with respect to age, sex, and ethnicity.[27] The database was established in 1987 and is a collaborative project between the National Institute for Health Research (NIHR) and the Medicines and Healthcare Regulatory Agency (MHRA). The information in the database comes from participating general practitioners (GPs), who are trained on recording medical information using standard software and coding systems. Medical diagnoses, referrals to specialists and secondary care settings, prescriptions, diagnostic testing, lifestyle information, and demographic data are all part of the recorded information.[28] Many validation studies have been performed that demonstrate the high quality of CPRD GOLD data.[28-30] The validity of the diagnoses of T2DM and VTE has been shown previously.[31-33]

\section{Study population}

In order to ensure that we only included incident DM cases in the study population, patients had to have a minimum of 3 years of DM-free history in the database prior to onset. We identified patients based on specific codes for T2DM. We also included patients with an unspecific code for DM (e.g. general code for "diabetes") if they were older than 30 years at diagnosis and received an oral antidiabetic drug (OAD). Independently of age, if DM patients never received insulin, we classified them as T2DM patients. We used the onset of DM as the study entry date, defined as the date of the first recorded DM code or the date of the first prescription for a DM medication. If the prescription occurred more than 365 days prior to the first recording of a DM diagnosis code, we excluded the patient.

We excluded patients with a diagnosis of cancer (except non-melanoma skin cancer), alcoholism, or HIV at any time in the patient record to avoid substantial bias and confounding.

We excluded patients with a history of VTE (at any time prior to the diagnosis of T2DM), or a code for surgery, immobilization, trauma, or paralysis and paresis within 3 months prior to the index date. We further excluded patients with a code for pregnancy or puerperium within 12 months prior to the index date.

\section{Case and control definition}

We defined cases as patients with a first-time recording of VTE during the study period, who received at least one prescription for an antithrombotic drug within 7 days prior until 90 days after the VTE,[5, 34, 35] including antiplatelet drugs, vitamin $\mathrm{K}$ antagonists, heparins, direct factor Xa inhibitors, direct thrombin inhibitors, fibrinolytic enzymes, or the synthetic penta-saccharide factor Xa inhibitor fondaparinux. The index date for each case was the date of the first recorded VTE. Since we excluded patients with known 
risk factors for a VTE prior to the outcome, we regard the VTE cases included in this study as having an unprovoked or idiopathic VTE.[5]

We used risk set sampling to match each case to 4 controls from the study population, i.e. patients who did not experience a VTE between the onset of DM and the index date of their matched case. We matched controls to cases on age ( $+/-3$ years), sex, general practice, index date (same index date as the case, and the control had to be present in the database on the index date), and T2DM duration (+/- 365 days assessed by counting the days between the study entry date and the index date).

\section{Exposure definition}

The exposure of interest in this study was glycemic control after the onset of DM defined by HbA1c levels. We used the last recorded $\mathrm{HbA} 1 \mathrm{c}$ value before the index date for our analyses. We assessed HbA1c levels in 7 categories: $\leq 6.5 \%$ ( $\leq 48 \mathrm{mmol} / \mathrm{mol})$, > 6.5-7.0\% (> 48-53 $\mathrm{mmol} / \mathrm{mol}$, reference group), $>7.0-7.5 \%$ (> 53-58 mmol $/ \mathrm{mol}$ ), > 7.5-8.0\% (> 58-64 mmol $/ \mathrm{mol}$ ), > 8.0-9.0\% (>64-75 mmol $/ \mathrm{mol}),>9.0 \%$ ( $>75 \mathrm{mmol} / \mathrm{mol})$, and no HbA1c measurement. Results for patients with missing values were presented in a separate category.

\section{Statistical analysis}

We used conditional logistic regression to assess the association between levels of glycemic control (expressed as $\mathrm{HbA} 1 \mathrm{c}$ levels) with $\mathrm{HbA} 1 \mathrm{c}$ levels of $>6.5-7.0 \%$ (> 48-53 mmol/mol) as the reference group and the risk of VTE, expressed as odds ratios (ORs) or adjusted ORs (aORs) with $95 \%$ confidence intervals (CI). We also assessed the association between HbA1c level and the risk of VTE according to the patients' number of GP visits during the study period. Lastly, we conducted analyses in men and women separately.

We adjusted for the following comorbidities and co-medications (recorded at any time in the patient record before the index date) in the final model based on previous clinical knowledge: BMI (categorical variable), smoking status (current, past, non-smokers, and unknown), CVD (including congestive heart failure, ischemic heart disease, myocardial infarction, hypertension, stroke), osteoarthritis, use of insulin, bisphosphonates, systemic corticosteroids, low-dose acetylsalicylic acid, and current (last prescription within 30 before the index date) or past (last prescription > 30 days prior to index date) use of metformin or sulfonylureas. We additionally adjusted for use of hormone replacement therapy (HRT) in a model restricted to women.

In sensitivity analyses, we 1) restricted the sample to patients whose last HbA1c measurement was recorded within less than 90 days prior to the index date, 2) analyzed the risk of VTE separately for patients with a previous CVD diagnosis, and 3) conducted separate analyses of the risk of VTE by HbA1c levels for patient groups of different T2DM durations.

We conducted analyses using SAS software version 9.4 (SAS Institute, Inc., Cary, NC, USA). 


\section{Results}

Within a cohort of 232'860 patients with incident T2DM who fulfilled all study inclusion and exclusion criteria, we identified 3'896 T2DM patients with an incident VTE diagnosis and 15'584 matched control patients (Fig. 1).

Cases and controls were similar with respect to age and time from most recent $\mathrm{HbA} 1 \mathrm{C}$ value to index date. We observed a mean of $12.2 \mathrm{HbA} 1 \mathrm{c}$ measurements per case and $11.9 \mathrm{HbA} 1 \mathrm{c}$ measurements per control during the study period. The median time between the index date and the last $\mathrm{HbA} 1 \mathrm{c}$ measurement was 115 days for cases and 116 for controls.

Patients exposed to insulin (aOR 1.58, 95\% $\mathrm{Cl}$ 1.37-1.81) had an increased risk of VTE compared to never-users of insulin, independently of HbA1c levels. However, cases and controls who had at least one prescription for insulin also had longer mean T2DM duration than non-users of these drugs (approximately 4.2 years longer). Table 1 provides information on the basic characteristics of cases and their matched controls at the index date. 
Table 1

Characteristics of the included cases and controls

\begin{tabular}{|c|c|c|c|c|}
\hline Characteristics & $\begin{array}{l}\text { Number of } \\
\text { cases (\%) }\end{array}$ & $\begin{array}{l}\text { Number of } \\
\text { controls (\%) }\end{array}$ & $\begin{array}{l}\text { Unadjusted } \\
\text { ORs } \\
(95 \% \mathrm{Cl})\end{array}$ & $\begin{array}{l}\text { Adjusted } \\
\text { ORs* } \\
(95 \% \mathrm{Cl})\end{array}$ \\
\hline \multicolumn{5}{|l|}{ Age (years) } \\
\hline$<60$ & 749 (19.2) & 3005 (19.3) & NA & NA \\
\hline $60-69$ & $878(22.5)$ & $3529(22.7)$ & NA & NA \\
\hline $70-79$ & 1295 (33.2) & $5180(33.2)$ & NA & NA \\
\hline $80+$ & $974(25.0)$ & $3870(24.8)$ & NA & NA \\
\hline \multicolumn{5}{|l|}{ Sex } \\
\hline Male & $1719(44.1)$ & $6876(44.1)$ & NA & NA \\
\hline Female & 2177 (55.9) & 8708 (55.9) & NA & NA \\
\hline \multicolumn{5}{|l|}{ BMI $\left(\mathrm{kg} / \mathrm{m}^{2}\right)$} \\
\hline$<18.5$ & $31(0.8)$ & $132(0.9)$ & $\begin{array}{l}1.17(0.81- \\
1.68)\end{array}$ & $\begin{array}{l}1.05(0.71- \\
1.54)\end{array}$ \\
\hline 18.5 to $<25.0$ & $502(12.9)$ & $2596(16.7)$ & 1 (reference) & 1 (reference) \\
\hline 25.0 to $<30.0$ & $1125(28.9)$ & $5449(35.0)$ & $\begin{array}{l}1.11(0.99- \\
1.23)\end{array}$ & $\begin{array}{l}1.08(0.97- \\
1.20)\end{array}$ \\
\hline 30.0 to $<35.0$ & $1093(28.1)$ & 4059 (26.1) & $\begin{array}{l}1.48(1.33- \\
1.66)\end{array}$ & $\begin{array}{l}1.42(1.28- \\
1.59)\end{array}$ \\
\hline 35.0 to $<40.0$ & $583(15.0)$ & 1854 (11.9) & $\begin{array}{l}1.80(1.59- \\
2.05)\end{array}$ & $\begin{array}{l}1.64(1.45- \\
1.87)\end{array}$ \\
\hline$\geq 40.0$ & $476(12.2)$ & $1105(7.1)$ & $\begin{array}{l}2.58(2.25- \\
2.95)\end{array}$ & $\begin{array}{l}2.30(1.99- \\
2.64)\end{array}$ \\
\hline Unknown & $86(2.2)$ & $389(2.5)$ & $\begin{array}{l}1.14(0.90- \\
1.45)\end{array}$ & $\begin{array}{l}1.17(0.90- \\
1.52)\end{array}$ \\
\hline \multicolumn{5}{|l|}{ Smoking status } \\
\hline Non-smoker & $1463(37.6)$ & $6484(41.6)$ & 1 (reference) & 1 (reference) \\
\hline Current smoker & $447(11.5)$ & $1813(11.6)$ & $\begin{array}{l}1.10(0.99- \\
1.22)\end{array}$ & $\begin{array}{l}1.04(0.93- \\
1.16)\end{array}$ \\
\hline \multicolumn{5}{|c|}{$\begin{array}{l}\text { * Adjusted for BMI (categorical), smoking (categorical), CHF, IHD, MI, stroke, hypertension, } \\
\text { osteoarthritis, and use of insulin, bisphosphonate, systemic corticosteroids, low-dose acetylsalicylic } \\
\text { acid, current and past use of metformin, and current and past use of sulfonylureas }\end{array}$} \\
\hline ** Use of other $n$ & possible & & & \\
\hline
\end{tabular}




\begin{tabular}{|c|c|c|c|c|}
\hline Characteristics & $\begin{array}{l}\text { Number of } \\
\text { cases (\%) }\end{array}$ & $\begin{array}{l}\text { Number of } \\
\text { controls (\%) }\end{array}$ & $\begin{array}{l}\text { Unadjusted } \\
\text { ORs } \\
(95 \% \mathrm{Cl})\end{array}$ & $\begin{array}{l}\text { Adjusted } \\
\text { ORs* } \\
(95 \% \mathrm{Cl})\end{array}$ \\
\hline Ex-smoker & $1927(49.5)$ & $7054(45.3)$ & $\begin{array}{l}1.23(1.15- \\
1.32)\end{array}$ & $\begin{array}{l}1.10(1.02- \\
1.18)\end{array}$ \\
\hline Unknown & $59(1.5)$ & $233(1.5)$ & $\begin{array}{l}1.10(0.84- \\
1.44)\end{array}$ & $\begin{array}{l}1.17(0.87- \\
1.57)\end{array}$ \\
\hline \multicolumn{5}{|c|}{ No. of $\mathrm{HbA} 1 \mathrm{c}$ measurements in the medical history before the index date } \\
\hline $1-4$ & $1402(36.0)$ & $5937(38.1)$ & 1 (reference) & 1 (reference) \\
\hline $5-9$ & $1638(42.0)$ & $6470(41.5)$ & $\begin{array}{l}1.10(1.02- \\
1.19)\end{array}$ & $\begin{array}{l}1.07(0.99- \\
1.16)\end{array}$ \\
\hline 10 or more & $518(13.3)$ & $1896(12.2)$ & $\begin{array}{l}1.22(1.09- \\
1.37)\end{array}$ & $\begin{array}{l}1.09(0.96- \\
1.23)\end{array}$ \\
\hline No Recording & $338(8.7)$ & $1281(8.2)$ & $\begin{array}{l}1.13(1.00- \\
1.29)\end{array}$ & $\begin{array}{l}1.18(1.03- \\
1.35)\end{array}$ \\
\hline \multicolumn{5}{|l|}{ Comorbidities } \\
\hline $\begin{array}{l}\text { Inflammatory bowel } \\
\text { disease }\end{array}$ & $148(3.8)$ & $310(2.0)$ & $\begin{array}{l}1.94(1.64- \\
2.30)\end{array}$ & $\begin{array}{l}1.69(1.41- \\
2.03)\end{array}$ \\
\hline Chronic renal failure & $296(7.6)$ & $844(5.4)$ & $\begin{array}{l}1.49(1.34- \\
1.69)\end{array}$ & $\begin{array}{l}1.22(1.06- \\
1.39)\end{array}$ \\
\hline Diabetic retinopathy & $1161(29.8)$ & $4330(27.8)$ & $\begin{array}{l}1.15(1.06- \\
1.25)\end{array}$ & $\begin{array}{l}1.15(1.05- \\
1.24)\end{array}$ \\
\hline Asthma & $817(21.0)$ & $2452(15.7)$ & $\begin{array}{l}1.43(1.33- \\
1.55)\end{array}$ & $\begin{array}{l}1.08(0.99- \\
1.18)\end{array}$ \\
\hline $\begin{array}{l}\text { Congestive heart failure } \\
\text { (CHF) }\end{array}$ & $444(11.4)$ & $1038(6.7)$ & $\begin{array}{l}1.86(1.68- \\
2.07)\end{array}$ & $\begin{array}{l}1.31(1.17- \\
1.47)\end{array}$ \\
\hline $\begin{array}{l}\text { Ischemic heart disease } \\
\text { (IHD) }\end{array}$ & $1192(30.6)$ & $3168(20.3)$ & $\begin{array}{l}1.79(1.66- \\
1.92)\end{array}$ & $\begin{array}{l}1.47(1.35- \\
1.60)\end{array}$ \\
\hline $\begin{array}{l}\text { Myocardial infarction } \\
\text { (Ml) }\end{array}$ & $481(12.4)$ & $1418(9.1)$ & $\begin{array}{l}1.43(1.29- \\
1.58)\end{array}$ & $\begin{array}{l}0.97(0.87- \\
1.09)\end{array}$ \\
\hline Stroke & $566(14.5)$ & $1652(10.6)$ & $\begin{array}{l}1.45(1.32- \\
1.59)\end{array}$ & $\begin{array}{l}1.37(1.24- \\
1.51)\end{array}$ \\
\hline Arterial hypertension & $2579(66.2)$ & $10180(65.3)$ & $\begin{array}{l}1.04(0.97- \\
1.12)\end{array}$ & $\begin{array}{l}0.96(0.90- \\
1.04)\end{array}$ \\
\hline \multicolumn{5}{|c|}{$\begin{array}{l}\text { * Adjusted for BMI (categorical), smoking (categorical), CHF, IHD, MI, stroke, hypertension, } \\
\text { osteoarthritis, and use of insulin, bisphosphonate, systemic corticosteroids, low-dose acetylsalicylic } \\
\text { acid, current and past use of metformin, and current and past use of sulfonylureas }\end{array}$} \\
\hline \multicolumn{5}{|c|}{$\star \star$ Use of other medication possible } \\
\hline
\end{tabular}




\begin{tabular}{|c|c|c|c|c|}
\hline Characteristics & $\begin{array}{l}\text { Number of } \\
\text { cases (\%) }\end{array}$ & $\begin{array}{l}\text { Number of } \\
\text { controls (\%) }\end{array}$ & $\begin{array}{l}\text { Unadjusted } \\
\text { ORs } \\
(95 \% \mathrm{Cl})\end{array}$ & $\begin{array}{l}\text { Adjusted } \\
\text { ORs* } \\
(95 \% \mathrm{Cl})\end{array}$ \\
\hline $\begin{array}{l}\text { Peripheral arterial } \\
\text { disease }\end{array}$ & $209(5.4)$ & $461(3.0)$ & $\begin{array}{l}1.87(1.61- \\
2.16)\end{array}$ & $\begin{array}{l}1.50(1.28- \\
1.76)\end{array}$ \\
\hline Osteoarthritis & $1480(38.0)$ & $4462(28.6)$ & $\begin{array}{l}1.60(1.49- \\
1.71)\end{array}$ & $\begin{array}{l}1.42(1.32- \\
1.53)\end{array}$ \\
\hline Rheumatoid arthritis & $124(3.2)$ & $299(1.9)$ & $\begin{array}{l}1.68(1.40- \\
2.02)\end{array}$ & $\begin{array}{l}1.31(1.07- \\
1.60)\end{array}$ \\
\hline Hyperlipidemia & $1060(27.2)$ & $3947(25.3)$ & $\begin{array}{l}1.11(1.03- \\
1.20)^{(10}\end{array}$ & $\begin{array}{l}0.99(0.92- \\
1.07)\end{array}$ \\
\hline Cardiovascular disease & $3116(80.0)$ & $11^{\prime} 673(74.9)$ & $\begin{array}{l}1.39(1.28- \\
1.51)\end{array}$ & $\begin{array}{l}1.10(0.96- \\
1.25)\end{array}$ \\
\hline \multicolumn{5}{|l|}{ Co-medication ** } \\
\hline Insulin & $493(12.7)$ & $1190(7.6)$ & $\begin{array}{l}1.90(1.68- \\
2.14)\end{array}$ & $\begin{array}{l}1.58(1.37- \\
1.81)\end{array}$ \\
\hline Glitazones & $538(13.8)$ & $1804(11.6)$ & $\begin{array}{l}1.28(1.14- \\
1.44)\end{array}$ & $\begin{array}{l}1.10(0.97- \\
1.24)\end{array}$ \\
\hline Sulfonylurea & $1482(38.0)$ & $5421(34.8)$ & $\begin{array}{l}1.19(1.10- \\
1.29)\end{array}$ & $\begin{array}{l}1.07(0.97- \\
1.18)\end{array}$ \\
\hline Metformin & $2396(61.5)$ & $9508(61.0)$ & $\begin{array}{l}1.03(0.95- \\
1.11)\end{array}$ & $\begin{array}{l}0.96 \\
(0.67 .1 .37)\end{array}$ \\
\hline GLP1 & $109(2.8)$ & $329(2.1)$ & $\begin{array}{l}1.45(1.14- \\
1.83)\end{array}$ & $\begin{array}{l}0.94(0.73- \\
1.21)\end{array}$ \\
\hline DPP4 & $314(8.1)$ & $1252(8.0)$ & $\begin{array}{l}1.00(0.87- \\
1.16)\end{array}$ & $\begin{array}{l}0.88(0.76- \\
1.03)\end{array}$ \\
\hline SGLT2 & $56(1.4)$ & $252(1.6)$ & $\begin{array}{l}0.87(0.64- \\
1.19)\end{array}$ & $\begin{array}{l}0.78(0.57- \\
1.09)\end{array}$ \\
\hline All oral antidiabetics & $2671(68.6)$ & $10512(67.5)$ & $\begin{array}{l}1.06(0.98- \\
1.16)\end{array}$ & $\begin{array}{l}1.05(0.89- \\
1.24)\end{array}$ \\
\hline Statins & $2989(76.7)$ & $11865(76.1)$ & $\begin{array}{l}1.04(0.95- \\
1.15)\end{array}$ & $\begin{array}{l}0.85(0.77- \\
0.94)\end{array}$ \\
\hline Bisphosphonates & $468(12.0)$ & $1225(7.9)$ & $\begin{array}{l}1.67(1.48- \\
1.88)\end{array}$ & $\begin{array}{l}1.38(1.21- \\
1.56)\end{array}$ \\
\hline
\end{tabular}

* Adjusted for BMI (categorical), smoking (categorical), CHF, IHD, MI, stroke, hypertension, osteoarthritis, and use of insulin, bisphosphonate, systemic corticosteroids, low-dose acetylsalicylic acid, current and past use of metformin, and current and past use of sulfonylureas

** Use of other medication possible 


\begin{tabular}{|c|c|c|c|c|}
\hline Characteristics & $\begin{array}{l}\text { Number of } \\
\text { cases (\%) }\end{array}$ & $\begin{array}{l}\text { Number of } \\
\text { controls (\%) }\end{array}$ & $\begin{array}{l}\text { Unadjusted } \\
\text { ORs } \\
(95 \% \mathrm{Cl})\end{array}$ & $\begin{array}{l}\text { Adjusted } \\
\text { ORs* } \\
(95 \% \mathrm{Cl})\end{array}$ \\
\hline Contraceptive pill & $75(1.9)$ & $357(2.3)$ & $\begin{array}{l}0.76(0.56- \\
1.04)\end{array}$ & $\begin{array}{l}0.81(0.59- \\
1.11)\end{array}$ \\
\hline $\begin{array}{l}\text { Hormone replacement } \\
\text { therapy }\end{array}$ & $656(16.8)$ & $2414(15.5)$ & $\begin{array}{l}1.15(1.03- \\
1.28)\end{array}$ & $\begin{array}{l}1.05(0.94- \\
1.18)\end{array}$ \\
\hline $\begin{array}{l}\text { Corticosteroids } \\
\text { (systemic) }\end{array}$ & $1409(36.2)$ & $3760(24.1)$ & $\begin{array}{l}1.83(1.69- \\
1.97)\end{array}$ & $\begin{array}{l}1.55(1.43- \\
1.69)\end{array}$ \\
\hline Coronary vasodilators & $1502(38.6)$ & $4019(25.8)$ & $\begin{array}{l}1.86(1.73- \\
2.01)\end{array}$ & $\begin{array}{l}1.36(1.23- \\
1.51)\end{array}$ \\
\hline $\begin{array}{l}\text { Low dose acetylsalicylic } \\
\text { acid }\end{array}$ & $2346(60.2)$ & $7929(50.9)$ & $\begin{array}{l}1.52(1.41- \\
1.64)\end{array}$ & $\begin{array}{l}1.35(1.25- \\
1.47)\end{array}$ \\
\hline Loop diuretics & $1701(43.7)$ & $4205(27.0)$ & $\begin{array}{l}2.28(2.11- \\
2.47)\end{array}$ & $\begin{array}{l}1.65(1.52- \\
1.81)\end{array}$ \\
\hline All diuretics & $2773(71.2)$ & $9395(60.3)$ & $\begin{array}{l}1.79(1.64- \\
1.94)\end{array}$ & $\begin{array}{l}1.47(1.33- \\
1.61)\end{array}$ \\
\hline \multicolumn{5}{|c|}{$\begin{array}{l}\text { * Adjusted for BMI (categorical), smoking (categorical), CHF, IHD, MI, stroke, hypertension, } \\
\text { osteoarthritis, and use of insulin, bisphosphonate, systemic corticosteroids, low-dose acetylsalicylic } \\
\text { acid, current and past use of metformin, and current and past use of sulfonylureas }\end{array}$} \\
\hline \multicolumn{5}{|c|}{$\star \star$ Use of other medication possible } \\
\hline
\end{tabular}

We found no elevated relative risk for VTE in patients with the last $\mathrm{HbA} 1 \mathrm{c}$ measurement $>7.0 \%(>53$ $\mathrm{mmol} / \mathrm{mol}$ ) compared to the reference group of patients with $\mathrm{HbA} 1 \mathrm{c}$ levels $>6.5-7.0 \%(>48-53$ $\mathrm{mmol} / \mathrm{mol}$ ). The ORs for the various $\mathrm{HbA} 1 \mathrm{c}$ categories are displayed in Table 2. There was no consistent linear increase in the risk of developing VTE with increasing HbA1c levels. Patients with missing HbA1c measurements had the highest risk of VTE $(\mathrm{aOR} 1.34,95 \% \mathrm{Cl} 1.14-1.57)$ when compared to patients with last $\mathrm{HbA} 1 \mathrm{c}$ measurements of $>6.5-7.0 \%(>48-53 \mathrm{mmol} / \mathrm{mol})$ before the index date. The majority of the cases with no HbA1c measurements (7.4\% in total) also had little GP contact ( $0-14$ GP visits: $22.9 \%$ ( $n=$ $100), 15-29$ visits: $6.6 \%(n=59), 15-29$ visits: $6.6 \%(n=59)$, and $30+$ visits: $5.1 \%(n=130)$.

When we stratified our analyses by sex (Table 2), we observed a slightly higher risk of VTE in women with $\mathrm{HbA1c}$ levels $>7.0 \%$ (> $53 \mathrm{mmol} / \mathrm{mol}$ ) compared to the reference group of women with HbA1c levels $>6.5$ $7.0 \%$ (> 48-53 mmol/mol). There was no association of HbA1c levels with risk of VTE in men. 
Table 2

Risk of VTE by HbA1c level

\begin{tabular}{|c|c|c|c|c|}
\hline HbA1c-level & $\begin{array}{l}\text { Number of } \\
\text { cases (\%) }\end{array}$ & $\begin{array}{l}\text { Number of } \\
\text { controls (\%) }\end{array}$ & $\begin{array}{l}\text { Unadjusted } \\
\text { ORs } \\
(95 \% \mathrm{Cl})\end{array}$ & $\begin{array}{l}\text { Adjusted } \\
\text { ORs* } \\
(95 \% \mathrm{Cl})\end{array}$ \\
\hline \multicolumn{5}{|c|}{ HbA1c-Values (last measurement before the index date) and risk of VTE } \\
\hline$\leq 6.5 \%(\leq 48 \mathrm{mmol} / \mathrm{mol})$ & $1258(32.3)$ & $5523(35.4)$ & $\begin{array}{l}0.99(0.90- \\
1.09)\end{array}$ & $\begin{array}{l}0.98(0.89- \\
1.08)\end{array}$ \\
\hline $\begin{array}{l}>6.5-7.0 \%(>48-53 \\
\mathrm{mmol} / \mathrm{mol})\end{array}$ & $672(17.3)$ & $2925(18.8)$ & 1 (reference) & 1 (reference) \\
\hline $\begin{array}{l}>7.0-7.5 \%(>53-58 \\
\mathrm{mmol} / \mathrm{mol})\end{array}$ & $587(15.1)$ & $2213(14.2)$ & $\begin{array}{l}1.16(1.04- \\
1.30)\end{array}$ & $\begin{array}{l}1.13(1.01- \\
1.26)\end{array}$ \\
\hline $\begin{array}{l}>7.5-8.0 \% \text { (> 58-64 } \\
\mathrm{mmol} / \mathrm{mol})\end{array}$ & $322(8.3)$ & $1235(8.3)$ & $\begin{array}{l}1.15(1.01- \\
1.32)\end{array}$ & $\begin{array}{l}1.10(0.96- \\
1.27)\end{array}$ \\
\hline $\begin{array}{l}>8.0-9.0 \% \text { (>64-75 } \\
\mathrm{mmol} / \mathrm{mol})\end{array}$ & $371(9.5)$ & $1296(8.3)$ & $\begin{array}{l}1.27(1.12- \\
1.44)\end{array}$ & $\begin{array}{l}1.14(1.00- \\
1.30)\end{array}$ \\
\hline$>9.0 \%(>75 \mathrm{mmol} / \mathrm{mol}))$ & $397(10.2)$ & $1365(8.8)$ & $\begin{array}{l}1.31(1.15- \\
1.48)\end{array}$ & $\begin{array}{l}1.08(0.94- \\
1.23)\end{array}$ \\
\hline No Recording & $289(7.4)$ & $1027(6.6)$ & $\begin{array}{l}1.29(1.11- \\
1.51)\end{array}$ & $\begin{array}{l}1.34(1.14- \\
1.57)\end{array}$ \\
\hline
\end{tabular}

$\mathrm{HbA1c}$-Values (last measurement before the index date) and risk of VTE in women

\begin{tabular}{|c|c|c|c|c|}
\hline$\leq 6.5 \%(\leq 48 \mathrm{mmol} / \mathrm{mol})$ & $729(33.5)$ & $3186(36.6)$ & $\begin{array}{l}1.05(0.93- \\
1.19)\end{array}$ & $\begin{array}{l}1.04(0.91- \\
1.18)\end{array}$ \\
\hline $\begin{array}{l}>6.5-7.0 \%(>48-53 \\
\mathrm{mmol} / \mathrm{mol})\end{array}$ & $357(16.4)$ & $1643(18.9)$ & 1 (reference) & 1 (reference) \\
\hline $\begin{array}{l}>7.0-7.5 \%(>53-58 \\
\mathrm{mmol} / \mathrm{mol})\end{array}$ & $326(15.0)$ & $1212(13.9)$ & $\begin{array}{l}1.25(1.07- \\
1.45)\end{array}$ & $\begin{array}{l}1.22(1.04- \\
1.42)\end{array}$ \\
\hline $\begin{array}{l}>7.5-8.0 \%(>58-64 \\
\mathrm{mmol} / \mathrm{mol})\end{array}$ & $177(8.1)$ & $649(7.5)$ & $\begin{array}{l}1.28(1.07- \\
1.53)\end{array}$ & $\begin{array}{l}1.24(1.03- \\
1.50)\end{array}$ \\
\hline $\begin{array}{l}>8.0-9.0 \% \text { (>64-75 } \\
\mathrm{mmol} / \mathrm{mol})\end{array}$ & $190(8.7)$ & $704(8.1)$ & $\begin{array}{l}1.27(1.06- \\
1.51)\end{array}$ & $\begin{array}{l}1.14(0.95- \\
1.38)\end{array}$ \\
\hline$>9.0 \%(>75 \mathrm{mmol} / \mathrm{mol}))$ & $223(10.2)$ & $714(8.2)$ & $\begin{array}{l}1.49(1.25- \\
1.77)\end{array}$ & $\begin{array}{l}1.24(1.02- \\
1.49)\end{array}$ \\
\hline No Recording & $175(8.0)$ & $600(6.9)$ & $\begin{array}{l}1.44(1.17- \\
1.77)\end{array}$ & $\begin{array}{l}1.51(1.22- \\
1.86)\end{array}$ \\
\hline
\end{tabular}

*Adjusted for BMI (categorical), smoking (categorical), CHF, IHD, MI, stroke, hypertension, osteoarthritis, and use of insulin, bisphosphonate, systemic corticosteroids, low-dose acetylsalicylic acid, current and past use of metformin, and current and past use of sulfonylureas. In women we additionally adjusted for HRT use. 


\begin{tabular}{|c|c|c|c|c|}
\hline HbA1c-level & $\begin{array}{l}\text { Number of } \\
\text { cases (\%) }\end{array}$ & $\begin{array}{l}\text { Number of } \\
\text { controls (\%) }\end{array}$ & $\begin{array}{l}\text { Unadjusted } \\
\text { ORs } \\
(95 \% \mathrm{Cl})\end{array}$ & $\begin{array}{l}\text { Adjusted } \\
\text { ORs* } \\
(95 \% \mathrm{Cl})\end{array}$ \\
\hline \multicolumn{5}{|c|}{ HbA1c-Values (last measurement before the index date) and risk of VTE in men } \\
\hline$\leq 6.5 \%(\leq 48 \mathrm{mmol} / \mathrm{mol})$ & $529(30.8)$ & $2337(34.0)$ & $\begin{array}{l}0.92(0.80- \\
1.06)\end{array}$ & $\begin{array}{l}0.93(0.81- \\
1.07)\end{array}$ \\
\hline $\begin{array}{l}>6.5-7.0 \%(>48-53 \\
\mathrm{mmol} / \mathrm{mol})\end{array}$ & $315(18.3)$ & $1282(18.6)$ & 1 (reference) & 1 (reference) \\
\hline $\begin{array}{l}>7.0-7.5 \%(>53-58 \\
\mathrm{mmol} / \mathrm{mol})\end{array}$ & $261(15.2)$ & $1001(14.6)$ & $\begin{array}{l}1.07(0.91- \\
1.26)\end{array}$ & $\begin{array}{l}1.04(0.88- \\
1.23)\end{array}$ \\
\hline $\begin{array}{l}>7.5-8.0 \%(>58-64 \\
\mathrm{mmol} / \mathrm{mol})\end{array}$ & $145(8.4)$ & $586(8.5)$ & $\begin{array}{l}1.02(0.83- \\
1.24)\end{array}$ & $\begin{array}{l}0.97(0.79- \\
1.19)\end{array}$ \\
\hline $\begin{array}{l}>8.0-9.0 \%(>64-75 \\
\mathrm{mmol} / \mathrm{mol})\end{array}$ & $181(10.5)$ & $592(8.6)$ & $\begin{array}{l}1.27(1.05- \\
1.52)\end{array}$ & $\begin{array}{l}1.15(0.95- \\
1.40)\end{array}$ \\
\hline$>9.0 \%(>75 \mathrm{mmol} / \mathrm{mol}))$ & $174(10.1)$ & $651(9.5)$ & $\begin{array}{l}1.11(0.92- \\
1.35)\end{array}$ & $\begin{array}{l}0.93(0.76- \\
1.13)\end{array}$ \\
\hline No Recording & $114(6.6)$ & $427(6.2)$ & $\begin{array}{l}1.12(0.89- \\
1.42)\end{array}$ & $\begin{array}{l}1.16(0.90- \\
1.49)\end{array}$ \\
\hline \multicolumn{5}{|c|}{$\begin{array}{l}\text { *Adjusted for BMI (categorical), smoking (categorical), CHF, IHD, MI, stroke, hypertension, } \\
\text { osteoarthritis, and use of insulin, bisphosphonate, systemic corticosteroids, low-dose acetylsalicylic } \\
\text { acid, current and past use of metformin, and current and past use of sulfonylureas. In women we } \\
\text { additionally adjusted for HRT use. }\end{array}$} \\
\hline
\end{tabular}

Among patients with preexisting CVD (Table 3 ), individuals with $\mathrm{HbA1c}$ levels $>7.0 \%(>53 \mathrm{mmol} / \mathrm{mol})$ had a similar risk of VTE compared to patients with $\mathrm{HbA1c}$ levels between $>6.5-7.0 \%$ (> 48-53 $\mathrm{mmol} / \mathrm{mol}$ ). There was a slightly higher risk of VTE with increased HbA1c levels in women with CVD, but not in men. 
Table 3

Risk of VTE according to HbA1c in patients with CVD

\begin{tabular}{|c|c|c|c|c|}
\hline Characteristics & $\begin{array}{l}\text { Number of cases } \\
(\%)\end{array}$ & $\begin{array}{l}\text { Number of controls } \\
(\%)\end{array}$ & $\begin{array}{l}\text { Unadjusted } \\
\text { ORs } \\
(95 \% \mathrm{Cl})\end{array}$ & $\begin{array}{l}\text { Adjusted ORs* } \\
(95 \% \mathrm{Cl})\end{array}$ \\
\hline \multicolumn{5}{|c|}{ CVD and risk of VTE } \\
\hline$\leq 6.5 \%$ & $1037(33.3)$ & $4325(37.1)$ & $\begin{array}{l}0.98(0.89- \\
1.09)\end{array}$ & $\begin{array}{l}1.00(0.90- \\
1.11)\end{array}$ \\
\hline$>6.5-7.0 \%$ & $557(17.8)$ & $2286(19.6)$ & 1 (reference) & 1 (reference) \\
\hline$>7.0-7.5 \%$ & $476(15.3)$ & $1675(14.4)$ & $\begin{array}{l}1.20(1.06- \\
1.36)\end{array}$ & $\begin{array}{l}1.18(1.04- \\
1.33)^{(1.04}\end{array}$ \\
\hline$>7.5-8.0 \%$ & $262(8.4)$ & $903(7.7)$ & $\begin{array}{l}1.20(1.04- \\
1.40)\end{array}$ & $1.17(1.00-1.36)$ \\
\hline$>8.0-9.0 \%$ & $292(9.4)$ & $931(8.0)$ & $\begin{array}{l}1.27(1.10- \\
1.48)\end{array}$ & $\begin{array}{l}1.15(0.98- \\
1.34)^{(10-}\end{array}$ \\
\hline$>9.0 \%$ & $288(9.2)$ & $911(7.8)$ & $\begin{array}{l}1.32(1.13- \\
1.53)\end{array}$ & $1.08(0.92-$ \\
\hline No Recording & $204(6.6)$ & $642(5.5)$ & $\begin{array}{l}1.27(1.06- \\
1.53)\end{array}$ & $\begin{array}{l}1.29(1.06- \\
1.57)\end{array}$ \\
\hline \multicolumn{5}{|c|}{ HbA1c-Values (last measurement before the index date) and risk of VTE in women } \\
\hline$\leq 6.5 \%$ & $610(34.4)$ & $2537(37.9)$ & $\begin{array}{l}1.08(0.94- \\
1.23)\end{array}$ & $\begin{array}{l}1.08(0.94- \\
1.25)\end{array}$ \\
\hline$>6.5-7.0 \%$ & $301(17.0)$ & $1320(19.7)$ & 1 (reference) & 1 (reference) \\
\hline$>7.0-7.5 \%$ & $270(15.2)$ & $936(14.0)$ & $\begin{array}{l}1.31(1.12- \\
1.55)\end{array}$ & $\begin{array}{l}1.29(1.09- \\
1.53)\end{array}$ \\
\hline$>7.5-8.0 \%$ & $154(8.7)$ & $496(7.4)$ & $\begin{array}{l}1.36(1.12- \\
1.66)\end{array}$ & $\begin{array}{l}1.33(1.08- \\
1.64)\end{array}$ \\
\hline$>8.0-9.0 \%$ & $149(8.4)$ & $531(7.9)$ & $1.22(1.00-1.49)$ & $\begin{array}{l}1.09(0.88- \\
1.35)\end{array}$ \\
\hline$>9.0 \%$ & $163(9.2)$ & $493(7.4)$ & $\begin{array}{l}1.50(1.23- \\
1.84)\end{array}$ & $\begin{array}{l}1.24(0.99- \\
1.54)\end{array}$ \\
\hline No Recording & $127(7.2)$ & $381(5.7)$ & $\begin{array}{l}1.56(1.23- \\
1.98)\end{array}$ & $\begin{array}{l}1.63(1.27- \\
2.08)\end{array}$ \\
\hline
\end{tabular}

*Adjusted for BMI (categorical), smoking (categorical), CHF, IHD, MI, stroke, hypertension, osteoarthritis, and use of insulin, bisphosphonate, systemic corticosteroids, low-dose acetylsalicylic acid, current and past use of metformin, and current and past use of sulfonylureas. In women we additionally adjusted for HRT use. 


\begin{tabular}{|c|c|c|c|c|}
\hline Characteristics & $\begin{array}{l}\text { Number of cases } \\
\text { (\%) }\end{array}$ & $\begin{array}{l}\text { Number of controls } \\
(\%)\end{array}$ & $\begin{array}{l}\text { Unadjusted } \\
\text { ORs } \\
(95 \% \mathrm{Cl})\end{array}$ & $\begin{array}{l}\text { Adjusted ORs* } \\
\text { (95\% Cl) }\end{array}$ \\
\hline$\leq 6.5 \%$ & $427(31.8)$ & 1788 (35.9) & $\begin{array}{l}0.87(0.74- \\
1.02)\end{array}$ & $\begin{array}{l}0.90(0.76- \\
1.06)\end{array}$ \\
\hline$>6.5-7.0 \%$ & $256(19.1)$ & $966(19.4)$ & 1 (reference) & 1 (reference) \\
\hline$>7.0-7.5 \%$ & $206(15.4)$ & 739 (14.8) & $\begin{array}{l}1.06(0.88- \\
1.28)\end{array}$ & $\begin{array}{l}1.05(0.86- \\
1.27)\end{array}$ \\
\hline$>7.5-8.0 \%$ & $108(8.1)$ & 407 (8.2) & $\begin{array}{l}1.02(0.81- \\
1.28)\end{array}$ & $\begin{array}{l}0.98(0.78- \\
1.25)\end{array}$ \\
\hline$>8.0-9.0 \%$ & 143 (10.7) & $400(8.0)$ & $\begin{array}{l}1.34(1.08- \\
1.66)\end{array}$ & $\begin{array}{l}1.22(0.98- \\
1.53)\end{array}$ \\
\hline$>9.0 \%$ & $125(9.3)$ & $418(8.4)$ & $\begin{array}{l}1.11(0.88- \\
1.38)^{(08-}\end{array}$ & $\begin{array}{l}0.92(0.72- \\
1.16)\end{array}$ \\
\hline No Recording & $77(5.7)$ & $261(5.2)$ & $\begin{array}{l}0.95(0.71- \\
1.26)\end{array}$ & $\begin{array}{l}0.93(0.68- \\
1.28)\end{array}$ \\
\hline \multicolumn{5}{|c|}{$\begin{array}{l}\text { *Adjusted for BMI (categorical), smoking (categorical), CHF, IHD, MI, stroke, hypertension, } \\
\text { osteoarthritis, and use of insulin, bisphosphonate, systemic corticosteroids, low-dose acetylsalicylic } \\
\text { acid, current and past use of metformin, and current and past use of sulfonylureas. In women we } \\
\text { additionally adjusted for HRT use. }\end{array}$} \\
\hline
\end{tabular}

Also in an analysis restricted to patients with a last $\mathrm{HbA} 1 \mathrm{c}$ measurement within 90 days prior to the index date (Table 4), we only found a slight association between $\mathrm{HbA} 1 \mathrm{c}$ levels $>7.0 \%$ ( $>53 \mathrm{mmol} / \mathrm{mol}$ ) and risk of VTE in women. The risk of VTE among women with a 90-day HbA1c level above $7.0 \%(>53 \mathrm{mmol} / \mathrm{mol})$ increased around $18-35 \%$ as compared to those with $\mathrm{HbA} 1 \mathrm{c}$ levels $>6.5-7.0 \%(>48-53 \mathrm{mmol} / \mathrm{mol})$. 
Table 4

Risk of VTE according to HbA1c levels measured within 90 days prior to the index date (i.d.)

\begin{tabular}{|c|c|c|c|c|}
\hline $\begin{array}{l}\text { HbA1c level }<90 \text { days prior to } \\
\text { i.d. overall }\end{array}$ & $\begin{array}{l}\text { Number of } \\
\text { cases (\%) }\end{array}$ & $\begin{array}{l}\text { Number of } \\
\text { controls (\%) }\end{array}$ & $\begin{array}{l}\text { Unadjusted } \\
\text { ORs } \\
(95 \% \mathrm{Cl})\end{array}$ & $\begin{array}{l}\text { Adjusted } \\
\text { ORs* } \\
(95 \% \mathrm{Cl})\end{array}$ \\
\hline$\leq 6.5 \%$ & $414(10.6)$ & 1940 (12.5) & $\begin{array}{l}0.94(0.80- \\
1.09)\end{array}$ & $\begin{array}{l}0.93(0.80- \\
1.09)\end{array}$ \\
\hline$>6.5-7.0 \%$ & $262(6.7)$ & $1154(7.4)$ & $\begin{array}{l}1 \\
\text { (reference) }\end{array}$ & $\begin{array}{l}1 \\
\text { (reference) }\end{array}$ \\
\hline$>7.0-7.5 \%$ & $251(6.4)$ & $928(6.0)$ & $\begin{array}{l}1.20(1.01- \\
1.42)\end{array}$ & $\begin{array}{l}1.19(1.00- \\
1.42)\end{array}$ \\
\hline$>7.5-8.0 \%$ & $169(4.3)$ & $562(3.6)$ & $\begin{array}{l}1.34(1.10- \\
1.63)\end{array}$ & $\begin{array}{l}1.31(1.07- \\
1.60)\end{array}$ \\
\hline$>8.0-9.0 \%$ & $199(5.1)$ & $619(4.0)$ & $\begin{array}{l}1.43(1.18- \\
1.72)\end{array}$ & $\begin{array}{l}1.31(1.08- \\
1.58)\end{array}$ \\
\hline$>9.0 \%$ & $190(4.9)$ & $666(4.3)$ & $\begin{array}{l}1.27(1.05- \\
1.54)\end{array}$ & $\begin{array}{l}1.07(0.88- \\
1.30)\end{array}$ \\
\hline No Recording** & 2411 (61.9) & 9715 (62.3) & $\begin{array}{l}1.10(0.97- \\
1.25)\end{array}$ & $\begin{array}{l}1.07(0.94- \\
1.22)\end{array}$ \\
\hline \multirow[t]{2}{*}{$\begin{array}{l}\text { HbA1c level }<90 \text { days prior to } \\
\text { i.d. in women }\end{array}$} & $\begin{array}{l}\text { Number of } \\
\text { cases (\%) }\end{array}$ & $\begin{array}{l}\text { Number of } \\
\text { controls (\%) }\end{array}$ & $\begin{array}{l}\text { Unadjusted } \\
\text { ORs }\end{array}$ & $\begin{array}{l}\text { Adjusted } \\
\text { ORs* }\end{array}$ \\
\hline & & & $(95 \% \mathrm{Cl})$ & $(95 \% \mathrm{Cl})$ \\
\hline$\leq 6.5 \%$ & $241(11.1)$ & $1116(12.8)$ & $\begin{array}{l}0.97(0.79- \\
1.18)\end{array}$ & $\begin{array}{l}0.96(0.78- \\
1.19)\end{array}$ \\
\hline$>6.5-7.0 \%$ & $147(6.8)$ & $660(8.6)$ & $\begin{array}{l}1 \\
\text { (reference) }\end{array}$ & $\begin{array}{l}1 \\
\text { (reference) }\end{array}$ \\
\hline$>7.0-7.5 \%$ & $143(6.6)$ & $502(5.8)$ & $\begin{array}{l}1.28(1.02- \\
1.62)^{(1.02-}\end{array}$ & $\begin{array}{l}1.30(1.02- \\
1.65)\end{array}$ \\
\hline$>7.5-8.0 \%$ & $87(4.0)$ & $297(3.4)$ & $\begin{array}{l}1.33(1.02- \\
1.73)\end{array}$ & $\begin{array}{l}1.35(1.03- \\
1.77)\end{array}$ \\
\hline$>8.0-9.0 \%$ & $103(4.7)$ & 337 (3.9) & $\begin{array}{l}1.38(1.07- \\
1.78)\end{array}$ & $\begin{array}{l}1.27(0.97- \\
1.66)\end{array}$ \\
\hline
\end{tabular}

*Adjusted for BMI (categorical), smoking (categorical), CHF, IHD, MI, stroke, hypertension, osteoarthritis, and use of insulin, bisphosphonate, systemic corticosteroids, low-dose acetylsalicylic acid, current and past use of metformin, and current and past use of sulfonylureas. In women we additionally adjusted for HRT use.

$\star *$ Included are patients with missing $\mathrm{HbA} 1 \mathrm{c}$ measurements as well as those with a last $\mathrm{HbA} 1 \mathrm{c}$ level recorded $>90$ days prior to the index date 


\begin{tabular}{|c|c|c|c|c|}
\hline $\begin{array}{l}\text { HbA1c level }<90 \text { days prior to } \\
\text { i.d. overall }\end{array}$ & $\begin{array}{l}\text { Number of } \\
\text { cases }(\%)\end{array}$ & $\begin{array}{l}\text { Number of } \\
\text { controls (\%) }\end{array}$ & $\begin{array}{l}\text { Unadjusted } \\
\text { ORs } \\
(95 \% \mathrm{Cl})\end{array}$ & $\begin{array}{l}\text { Adjusted } \\
\text { ORs* } \\
(95 \% \mathrm{Cl})\end{array}$ \\
\hline$>9.0 \%$ & $112(5.1)$ & $358(4.1)$ & $\begin{array}{l}1.42(1.11- \\
1.83)\end{array}$ & $\begin{array}{l}1.18(0.91- \\
1.54)\end{array}$ \\
\hline No Recording** & 1344 (61.7) & $5438(62.5)$ & $\begin{array}{l}1.11(0.94- \\
1.32)\end{array}$ & $\begin{array}{l}1.09(0.91- \\
1.29)\end{array}$ \\
\hline \multirow[t]{2}{*}{$\begin{array}{l}\text { HbA1c level < } 90 \text { days prior to } \\
\text { i.d. in men }\end{array}$} & \multirow[t]{2}{*}{$\begin{array}{l}\text { Number of } \\
\text { cases }(\%)\end{array}$} & \multirow[t]{2}{*}{$\begin{array}{l}\text { Number of } \\
\text { controls (\%) }\end{array}$} & $\begin{array}{l}\text { Unadjusted } \\
\text { ORs }\end{array}$ & $\begin{array}{l}\text { Adjusted } \\
\text { ORs* }\end{array}$ \\
\hline & & & $(95 \% \mathrm{Cl})$ & $(95 \% \mathrm{Cl})$ \\
\hline$\leq 6.5 \%$ & $173(10.1)$ & $824(12.0)$ & $\begin{array}{l}0.90(0.71- \\
1.14)\end{array}$ & $\begin{array}{l}0.90(0.71- \\
1.14)\end{array}$ \\
\hline$>6.5-7.0 \%$ & 115 (6.7) & $494(7.2)$ & $\begin{array}{l}1 \\
\text { (reference) }\end{array}$ & $\begin{array}{l}1 \\
\text { (reference) }\end{array}$ \\
\hline$>7.0-7.5 \%$ & 108 (6.3) & $426(6.2)$ & $\begin{array}{l}1.09(0.84- \\
1.42)\end{array}$ & $\begin{array}{l}1.07(0.82- \\
1.40)\end{array}$ \\
\hline$>7.5-8.0 \%$ & $82(4.8)$ & 265 (3.9) & $\begin{array}{l}1.35(1.01- \\
1.80)\end{array}$ & $\begin{array}{l}1.27(0.95- \\
1.70)\end{array}$ \\
\hline$>8.0-9.0 \%$ & $96(5.6)$ & $282(4.1)$ & $\begin{array}{l}1.48(1.12- \\
1.94)\end{array}$ & $\begin{array}{l}1.34(1.02- \\
1.78)\end{array}$ \\
\hline$>9.0 \%$ & 78 (4.5) & $308(4.5)$ & $\begin{array}{l}1.10(0.82- \\
1.48)\end{array}$ & $\begin{array}{l}0.94(0.70- \\
1.27)\end{array}$ \\
\hline No Recording** & 1067 (62.1) & $4277(62.2)$ & $\begin{array}{l}1.08(0.89- \\
1.31)\end{array}$ & $\begin{array}{l}1.06(0.87- \\
1.29)\end{array}$ \\
\hline \multicolumn{5}{|c|}{$\begin{array}{l}\text { *Adjusted for BMI (categorical), smoking (categorical), CHF, IHD, MI, stroke, hypertension, } \\
\text { osteoarthritis, and use of insulin, bisphosphonate, systemic corticosteroids, low-dose acetylsalicylic } \\
\text { acid, current and past use of metformin, and current and past use of sulfonylureas. In women we } \\
\text { additionally adjusted for HRT use. }\end{array}$} \\
\hline \multicolumn{5}{|c|}{$\begin{array}{l}\star \star \text { Included are patients with missing } \mathrm{HbA} 1 \mathrm{c} \text { measurements as well as those with a last } \mathrm{HbA} 1 \mathrm{c} \text { level } \\
\text { recorded }>90 \text { days prior to the index date }\end{array}$} \\
\hline
\end{tabular}

We found no association between the risk of VTE by HbA1c level in the group of patients with a T2DM duration of more than 5 years (Table 5). However, among patients with shorter T2DM duration (0-5 years), those with $\mathrm{HbA} 1 \mathrm{c}$ levels $>7.0 \%$ (> $53 \mathrm{mmol} / \mathrm{mol}$ ) had slightly higher aORs for VTE when compared to T2DM patients with $\mathrm{HbA} 1 \mathrm{c}$ levels of $>6.5-7.0 \%$ (HbA1c > 7.0-7.5\%: aOR 1.25, 95\% Cl 1.06-1.47; $\mathrm{HbA} 1 \mathrm{c}$ > 7.5-8.0\%: aOR 1.21, 95\% Cl 0.98-1.50; HbA1c > 8.0-9.0\%: aOR 1.18, 95\% Cl 0.96-1.45; HbA1c > 9.0\%: aOR $1.18,95 \% \mathrm{Cl} 0.95-1.80)$. 
Table 5

Risk of VTE in patients with different T2DM durations

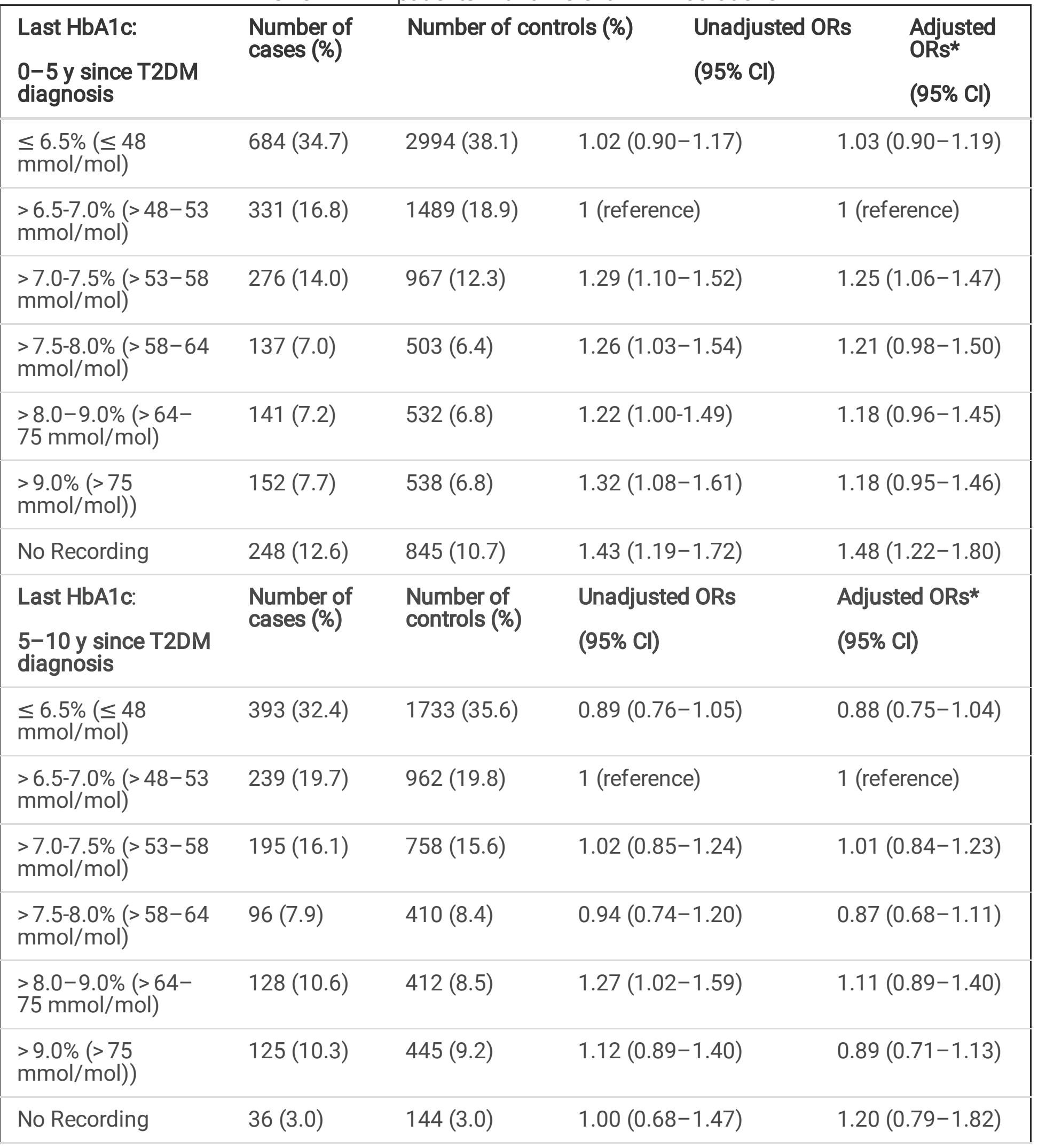

*Adjusted for BMI (categorical), smoking (categorical), CHF, IHD, MI, stroke, hypertension, osteoarthritis, and use of insulin, bisphosphonate, systemic corticosteroids, low-dose acetylsalicylic acid, current and past use of metformin, and current and past use of sulfonylureas. 


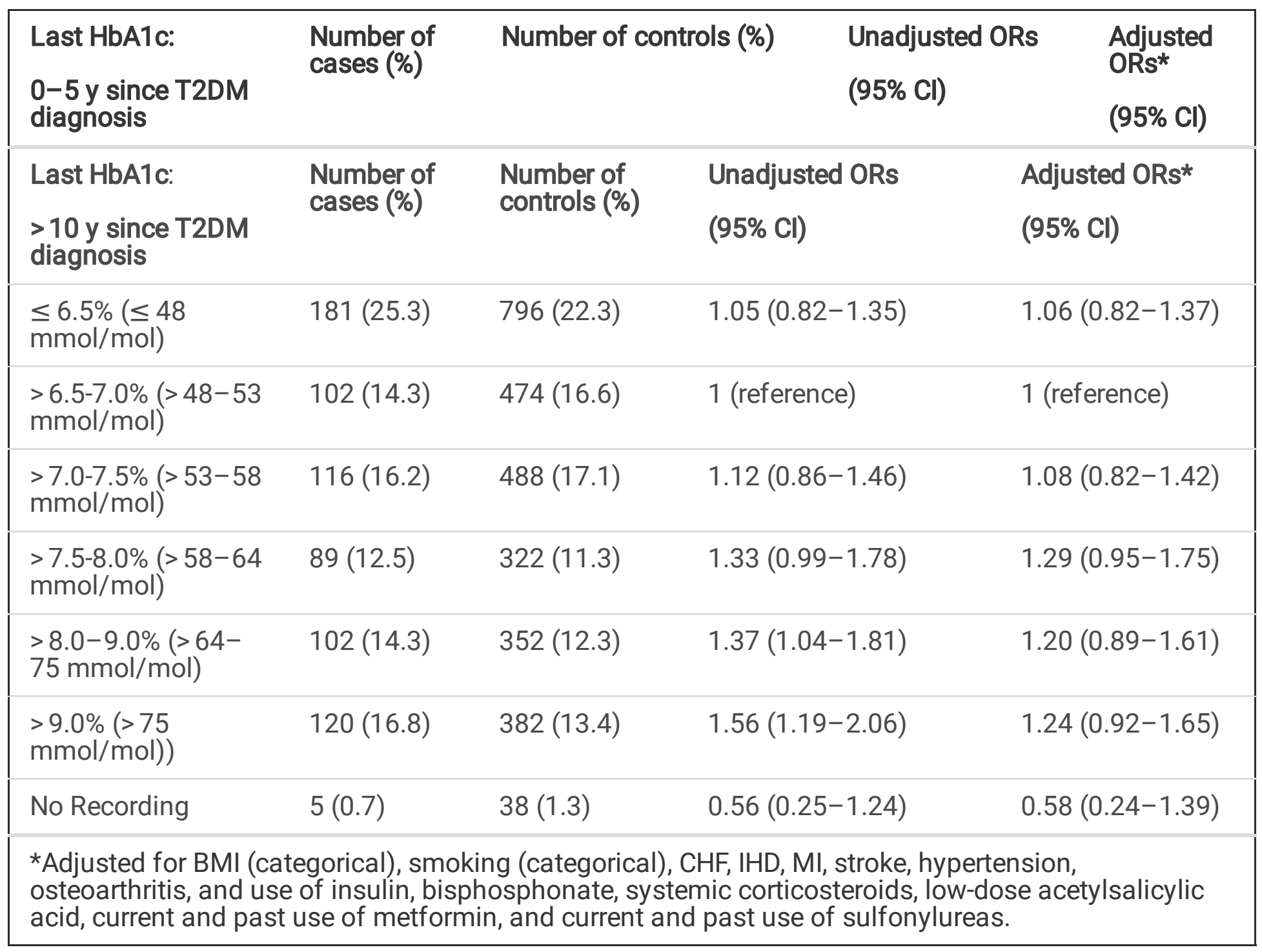

\section{Discussion}

In this large case-control study based on primary care data from the UK, patients with $\mathrm{HbA1c}>7.0 \%$ ( $>53$ $\mathrm{mmol} / \mathrm{mol}$ ) did not have an increased risk of unprovoked VTE compared to patients with $\mathrm{HbA} 1 \mathrm{c}>6.5$ $7.0 \%$ (> 48-53 mmol $/ \mathrm{mol})$. In the subset of female patients, we found a suggestion of a slightly increased risk of VTE in women with $\mathrm{HbA} 1 \mathrm{c}>7.0 \%$ (for example $\mathrm{HbA} 1 \mathrm{c}>7.5-8.0 \%$ : aOR $1.24,95 \% \mathrm{Cl}$ 1.03-1.50) when compared to those with $\mathrm{HbA} 1 \mathrm{c}>6.5-7.0 \%$ (> $48-53 \mathrm{mmol} / \mathrm{mol})$. This increase was slightly more pronounced if we only considered patients with $\mathrm{HbA} 1 \mathrm{c}$ measurements taken within 90 days prior to the index date. Overall, however, the association in women was weak, and there was no trend of increasing risk of VTE in association with increasing $\mathrm{HbA} 1 \mathrm{c}$ values. We did not observe an increased risk of VTE in men at any level of glycemic control.

The weak association between elevated $\mathrm{HbA} 1 \mathrm{c}$ levels and risk of VTE in women, but not in men, may be explained by the fact that pre-diabetic and diabetic women are more affected by chronically elevated cardiovascular risk factors, and their health declines faster when compared to men. [22, 36, 37]. Since T2DM is a disease with uncertain onset, which can remain undiagnosed for many years, this difference in 
risk factor levels between men and women is relevant. Several studies, including a comprehensive metaanalysis, suggest that the presence of diabetes eliminates the biological female advantage that is often used to explain the lower absolute rates of coronary heart disease (CHD) and stroke in women compared to men. $[22,38,39]$ The authors of this meta-analysis estimate that the relative risk for $\mathrm{CHD}$ is $44 \%$ greater in women with diabetes than in similarly affected men.[39] In general, our study population included more women than men, even though men are more often affected by T2DM and by VTE, when the diseases are observed independently of each other. The T2DM cohort for our study also included more men than women prior to the identification of the VTE cases (50.8\% vs $49.2 \%$ ). Several studies provide an explanation for this imbalance in the rates of affected females and males by showing that adverse changes in metabolic and vascular risk factor profiles are greater in women than in men. These changes occur in diabetic individuals as well as earlier in pre-diabetic individuals.[15, 22-24]

Patients with CVD and $\mathrm{HbA} 1 \mathrm{c}$ levels $>7.0 \%$ ( $>53 \mathrm{mmol} / \mathrm{mol})$ did not have an increased risk of VTE when compared to those with $\mathrm{HbA} 1 \mathrm{c}$ levels between $>6.5-7.0 \%$ (> 48-53 $\mathrm{mmol} / \mathrm{mol}$ ), though women with CVD and $\mathrm{HbA} 1 \mathrm{c}$ level $>7 \%$ (>53 mmol/mol) had a slightly elevated risk for VTE, while men with CVD did not. This result emphasizes the general importance of proper glycemic control in women suffering from both, CVD and T2DM.

In our study, patients with no recorded $\mathrm{HbA} 1 \mathrm{c}$ measurements had a higher risk of VTE compared to patients with $\mathrm{HbA} 1 \mathrm{c}>6.5-7.0 \%$ (> 48-53 $\mathrm{mmol} / \mathrm{mol}$ ) throughout our analyses. This could be a proxy for a lack of patient-doctor interaction and poor treatment adherence, which could lead not only to a higher risk for VTE (as suggested in this study), but potentially to other complications caused by improper management of T2DM.

The present findings should be interpreted within the context of the strengths and limitations of an observational study. A delayed diagnosis of T2DM may have led to the inclusion of some prevalent (instead of incident) T2DM cases in our cohort. Additionally, the UK Prospective Diabetes study found that a high prevalence of DM tissue damage was already present by the time the DM diagnosis was made, which is an indication of pre-existing DM.[40] Therefore, we may have underestimated the time until VTE events (after the recorded DM diagnosis) in our study population, which could have potentially affected our matching on DM duration. However, this misclassification is unlikely to have been differential by $\mathrm{HbA} 1 \mathrm{c}$ level, and we do not expect that it had a major influence on our findings.

Though VTE events are well recorded and have previously been validated in the CPRD (positive predictive value $88.2 \%$ [82.3-92.6\%] for VTE),[33] it is possible that we missed some unrecorded VTEs. This possible misclassification would likely be non-differential and would not materially change the results.

The strengths of our study include the large study sample and the observational nested case-control design within a cohort of patients with newly diagnosed T2DM. Our data come from a well validated primary care database that contains prospectively and routinely collected data, which avoids recall bias. Even though we only used the last $\mathrm{HbA} 1 \mathrm{c}$ measurement before the index date, $\mathrm{HbA} 1 \mathrm{c}$ measurements are regularly performed in the diabetic population, and median time between the index date and the last 
$\mathrm{HbA1c}$ measurement was short. This shows that the recorded $\mathrm{HbA1c}$ measurements provide a reliable and timely source for our analyses on the effect of glycemic control on the risk of VTE.

Our study population included a high proportion of patients with T2DM with $\mathrm{HbA} 1 \mathrm{c} \leq 7 \%$ ( $>53$ $\mathrm{mmol} / \mathrm{mol}$ ) who may have been healthier than the T2DM populations analyzed in other studies. Nevertheless, our population consisted of over 19'480 patients with T2DM, many of whom had HbA1c levels $>7 \%$ ( $>53 \mathrm{mmol} / \mathrm{mol})$. Therefore, we expect our results to be generalizable to those of other populations with T2DM and $\mathrm{HbA} 1 \mathrm{c}$ levels $>7 \%(>53 \mathrm{mmol} / \mathrm{mol})$.

In conclusion, our study provides evidence that $\mathrm{HbA1c}$ levels $>7 \%$ (>53 $\mathrm{mmol} / \mathrm{mol})$ are not associated with a materially increased risk for unprovoked VTE overall. There was a suggestion of a slightly increased VTE risk in women, which may be real or may reflect differences in lifestyle or other patient characteristics.

\section{Conclusion}

Our study raises the possibility that female T2DM patients with $\mathrm{HbA} 1 \mathrm{c}$ levels $>7 \%$ may have a slightly higher risk for unprovoked VTE compared to women with $\mathrm{HbA1}$ c level $>6.5-7.0 \%$. This increase may not be causal and may reflect differences in life style or other characteristics. We observed no effect of glycemic control on the risk of VTE in men.

\section{Abbreviations}




\begin{tabular}{|ll|}
\hline aOR & Adjusted Odds Ratio \\
\hline BMI & Body Mass Index \\
\hline CHD & Coronary Heart Disease \\
\hline CHF & Congestive Heart Failure \\
\hline Cl & Confidence Interval \\
\hline CPRD & Clinical Practice Research Datalink \\
\hline CVD & Cardiovascular Disease \\
\hline DM & Diabetes Mellitus \\
\hline DVT & Deep Vein Thrombosis \\
\hline GFAT & Glutamine Fructose-6 Phosphate Amidotransferase \\
\hline GP & General Practitioner \\
\hline HbA1c & Glycated Hemoglobin A1c \\
\hline HRT & Hormone Replacement Therapy \\
\hline i.d. & Index Date \\
\hline IHD & Ischemic Heart Disease \\
\hline ISAC & Independent Scientific Advisory Committee \\
\hline MHRA & Medicines And Healthcare Products Regulatory Agency \\
\hline MI & Myocardial infarction \\
\hline NHS & National Health Services \\
\hline NIHR & National Institute For Health Research \\
\hline OAD & Oral Antidiabetic Drug \\
\hline OR & Odds Ratio \\
\hline PE & Pulmonary Embolism \\
\hline UK & United Kingdom \\
\hline T1DM & Type 1 Diabetes Mellitus \\
\hline T2DM & Type 2 Diabetes Mellitus \\
\hline
\end{tabular}

\section{Declarations}




\section{Ethics approval and consent to participate}

The study protocol was approved by the Independent Scientific Advisory Committee (ISAC) for MHRA database research (protocol number 20_033) prior to the initiation of the study. The study protocol was made available to the journal reviewers.

\section{Consent for publication}

All of the authors have approved the contents of this paper and have agreed to the Cardiovascular Diabetology's submission policies.

\section{Availability of data and materials}

The data that support the findings of this study are available from the CPRD but restrictions apply to the availability of these data, which were used under license for the current study, and so are not publicly available. Data are however available from the authors upon reasonable request and with permission of CPRD.

\section{Competing interests}

We have no conflict of interest to declare, financial or otherwise.

\section{Funding}

Not applicable

\section{Authors' contributions}

Each of the named authors has substantially contributed to the preparation of this manuscript.

\section{Acknowledgements}

We thank Pascal Egger (Basel Pharmacoepidemiology Unit, University of Basel, Switzerland) for his technical support and programing.

This study is based in part on data from the Clinical Practice Research Datalink obtained under licence from the UK Medicines and Healthcare Products Regulatory Agency. The data is provided by patients and collected by the NHS as part of their care and support. The interpretation and conclusions contained in this study are those of the authors alone.

\section{References}

1. Cho $\mathrm{NH}$, et al. IDF Diabetes Atlas: Global estimates of diabetes prevalence for 2017 and projections for 2045. Diabetes Res Clin Pract. 2018;138:271-81. 
2. The British Diabetic Association. Diabetes UK. Us, diabetes and a lot of facts and stats. [Fact sheet] 2019. Last accessed: [15.04.2021]. Retrieved from:

https://www.diabetes.org.uk/professionals/position-statements-reports/statistics.

3. Dokken BB. The Pathophysiology of Cardiovascular Disease and Diabetes: Beyond Blood Pressure and Lipids. Diabetes Spectrum. 2008;21(3):160-5.

4. Carr ME. Diabetes mellitus: A hypercoagulable state. Journal of Diabetes its Complications. 2001;15(1):44-54.

5. National Institute for Health and Care Excellence (NICE). Venous thromboembolic diseases: diagnosis, management and thrombophilia testing (NG158). NICE Pathways; 2020.

6. Heit JA, et al., Is diabetes mellitus an independent risk factor for venous thromboembolism?: a population-based case-control study. Arteriosclerosis, thrombosis, and vascular biology, 2009. 29(9): p. $1399-1405$.

7. Silverstein MD, et al. Trends in the incidence of deep vein thrombosis and pulmonary embolism: a 25year population-based study. Arch Intern Med. 1998;158(6):585-93.

8. Essien E-O, Rali P, Mathai SC. Pulmonary Embolism. Med Clin North Am. 2019;103(3):549-64.

9. National Institute for Health and Care Excellence (NICE). Venous thromboembolic in adults: diagnosis and management (QS29). NICE Pathways. 2021. Last updated: [02.02.2021]. p. 5.

10. Martinez C, et al. Epidemiology of first and recurrent venous thromboembolism: a population-based cohort study in patients without active cancer. Thromb Haemost. 2014;112(2):255-63.

11. Engbers MJ, Van Hylckama A, Vlieg, Rosendaal FR. Venous thrombosis in the elderly: incidence, risk factors and risk groups. J Thromb Haemost. 2010;8(10):2105-12.

12. Tsai AW, et al. Cardiovascular Risk Factors and Venous Thromboembolism Incidence: The Longitudinal Investigation of Thromboembolism Etiology. Arch Intern Med. 2002;162(10):1182-9.

13. Lemkes BA, et al. Hyperglycemia: a prothrombotic factor? J Thromb Haemost. 2010;8(8):1663-9.

14. Petrauskiene $V$, et al. The risk of venous thromboembolism is markedly elevated in patients with diabetes. Diabetologia. 2005;48(5):1017-21.

15. Evans RW, Orchard TJ. Oxidized lipids in insulin-dependent diabetes mellitus: A sex-diabetes interaction? Metabolism. 1994;43(9):1196-200.

16. Kentner AC, Grace SL. Between mind and heart: Sex-based cognitive bias in cardiovascular disease treatment. Front Neuroendocrinol. 2017;45:18-24.

17. Selvin E, et al. Glycated Hemoglobin, Diabetes, and Cardiovascular Risk in Nondiabetic Adults. N Engl J Med. 2010;362(9):800-11.

18. Fuller $\mathrm{JH}$, et al., Mortality from coronary heart disease and stroke in relation to degree of glycaemia: the Whitehall study. British medical journal (Clinical research ed.), 1983. 287(6396): p. 867-870.

19. Bell EJ, et al. Glycemia (hemoglobin A1c) and incident venous thromboembolism in the Atherosclerosis Risk in Communities cohort study. Vascular Medicine. 2013;18(5):245-50. 
20. Hermanides $\mathrm{J}$, et al. Venous thrombosis is associated with hyperglycemia at diagnosis: a casecontrol study. J Thromb Haemost. 2009;7(6):945-9.

21. Lerstad G, et al. Hyperglycemia, assessed according to HbA1c, and future risk of venous thromboembolism: the Troms $\varnothing$ study. J Thromb Haemost. 2014;12(3):313-9.

22. Appelman $Y$, et al. Sex differences in cardiovascular risk factors and disease prevention. Atherosclerosis. 2015;241(1):211-8.

23. Howard BV, et al. Adverse Effects of Diabetes on Multiple Cardiovascular Disease Risk Factors in Women: The Strong Heart Study. Diabetes Care. 1998;21(8):1258-65.

24. Mansfield MW, Heywood DM, Grant PJ. Sex differences in coagulation and fibrinolysis in white subjects with non-insulin-dependent diabetes mellitus. Arterioscler Thromb Vasc Biol. 1996;16(1):160-4.

25. Laakso M. Heart in diabetes: a microvascular disease. Diabetes Care. 2011;34(Suppl 2):S145-9.. . Suppl 2.

26. Brownlee M. The Pathobiology of Diabetic Complications. A Unifying Mechanism. 2005;54(6):161525.

27. Mathur R, et al. Completeness and usability of ethnicity data in UK-based primary care and hospital databases. Journal of public health. 2013;36(4):684-92.

28. Herrett E, et al. Data resource profile: clinical practice research datalink (CPRD). Int J Epidemiol. 2015;44(3):827-36.

29. Jick H, Jick SS, Derby LE. Validation of information recorded on general practitioner based computerised data resource in the United Kingdom. Bmj. 1991;302(6779):766-8.

30. Jick SS, et al. Validity of the general practice research database. Pharmacotherapy: The Journal of Human Pharmacology Drug Therapy. 2003;23(5):686-9.

31. Meier CR, et al. HMG-CoA reductase inhibitors and the risk of fractures. Jama. 2000;283(24):320510.

32. Schlienger RG, et al. Use of $\beta$-blockers and risk of fractures. Jama. 2004;292(11):1326-32.

33. Khan NF, Harrison SE, Rose PW. Validity of diagnostic coding within the General Practice Research Database: a systematic review. Br J Gen Pract. 2010;60(572):e128-36.

34. Martín-Merino E, et al. Risk of venous thromboembolism among users of different anti-osteoporosis drugs: a population-based cohort analysis including over 200,000 participants from Spain and the UK. Osteoporos Int. 2018;29(2):467-78.

35. Ruigómez A, et al. Validation of venous thromboembolism diagnoses in patients receiving rivaroxaban or warfarin in The Health Improvement Network. Pharmacoepidemiol Drug Saf. 2021;30(2):229-36.

36. Donahue RP, et al., Sex Differences in Endothelial Function Markers Before Conversion to PreDiabetes: Does the Clock Start Ticking Earlier Among Women? The Western New York Study, 2007. 30(2): pp. 354-9. 
37. Haffner SM, Miettinen H, Stern MP. Relatively more atherogenic coronary heart disease risk factors in prediabetic women than in prediabetic men. Diabetologia. 1997;40(6):711-7.

38. Huxley R, Barzi F, Woodward M. Excess risk of fatal coronary heart disease associated with diabetes in men and women: meta-analysis of 37 prospective cohort studies. BMJ. 2006;332(7533):73-8.

39. Peters SAE, Huxley RR, Woodward M. Diabetes as risk factor for incident coronary heart disease in women compared with men: a systematic review and meta-analysis of 64 cohorts including 858,507 individuals and 28,203 coronary events. Diabetologia. 2014;57(8):1542-51.

40. Group UPDS. UK Prospective Diabetes Study XII: differences between Asian, Afro-Caribbean and white Caucasian type 2 diabetic patients at diagnosis of diabetes. Diabet Med. 1994;11(7):670-7.

\section{Figures}

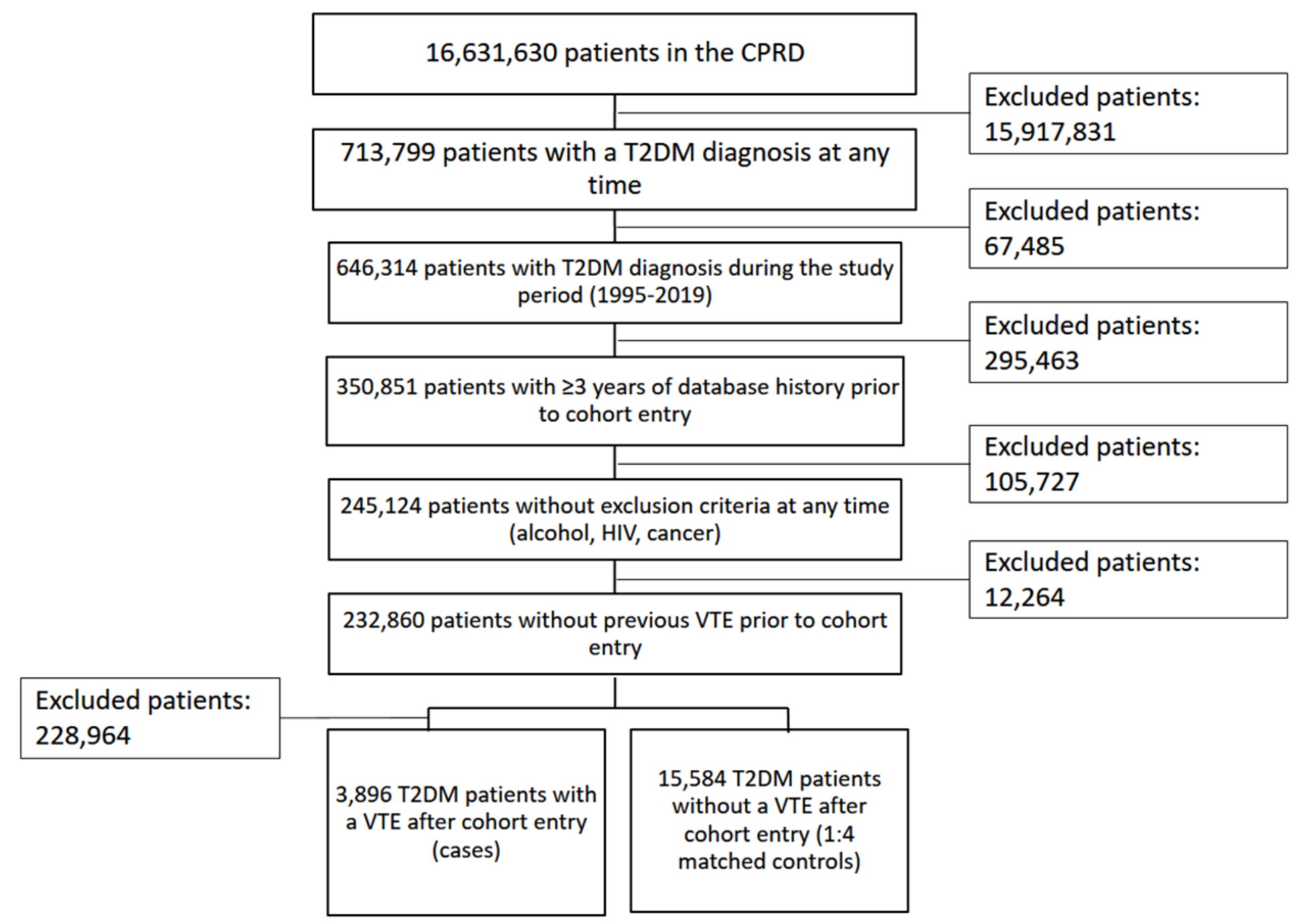

\section{Figure 1}

Selection of the study population 